Original

\title{
Dental anxiety and salivary cortisol levels before urgent dental care
}

\author{
Kazue Kanegane ${ }^{1)}$, Sibele S. Penha ${ }^{1)}$, Carolina D. Munhoz ${ }^{2)}$ and Rodney G. Rocha ${ }^{1)}$ \\ ${ }^{1)}$ Department of Stomatology, Faculty of Dentistry, University of São Paulo, São Paulo, Brazil \\ ${ }^{2}$ Institute of Biomedical Sciences, São Paulo, Brazil
}

(Received 23 March and accepted 7 July 2009)

\begin{abstract}
Dental anxiety is still prevalent, despite advances in treatment, and affects the utilization of health care services. The purpose of this cross-sectional study was to determine if patients with different degrees of dental anxiety and pain undergoing emergency dental care have different stress reactions as measured by salivary cortisol. Seventy three patients completed the modified dental anxiety scale (MDAS), and described any previous dental traumatic experience. Their socio-demographic characteristics were also recorded. They also rated pain intensity on a $100 \mathrm{~mm}$ visual analogue scale (VAS). A saliva sample was collected before the procedure, and analyzed by enzyme immunoassay. Thirty patients were dentally anxious and forty one complained of pain. In this sample, dental anxiety was not related to gender, age, educational level and family income; however, a previous traumatic event was related to dental anxiety. There was no association between salivary cortisol concentrations and gender or dental anxiety. Patients with pain showed higher cortisol levels. When gathering patient information, the dentist should note patients' negative dental experiences in order to provide more effective, less traumatic treatment. (J Oral Sci 51, 515-520, 2009)
\end{abstract}

Keywords: dental anxiety; urgency; pain; salivary cortisol.

Correspondence to Dr. Sibele Sarti Penha, Departamento de Estomatologia, Faculdade de Odontologia da Universidade de São Paulo, Av. Prof. Lineu Prestes, 2227, Cidade Universitária, São Paulo 05508-900, SP, Brasil

Tel: +55-11-3091-7893

Fax: +55-11-3091-7820

E-mail: sibsarti@usp.br

\section{Introduction}

Dental treatment may cause pain and discomfort. Even the expectation of pain increases dental anxiety, thus maintaining the number of dentally anxious persons (1).

The prevalence of dental anxiety has been the subject of many surveys. Dental anxiety and fear of pain associated with dentistry are relatively stable over time, despite advances in dental equipment, procedures and preventive measures. Thus, dental anxiety is a confounding problem with which dentists have to cope. Studies of the prevalence of dental anxiety in general population samples have reported estimates ranging from $4 \%$ (1) to $23.4 \%$ (2). The dental anxiety scale (DAS) is an instrument widely used in epidemiology and clinical research to assess the tendency to appraise dental treatment as dangerous and frightening (3). Humphris et al. (4) standardized the answering schemes and added a fifth item concerning anesthetic injection, which is of greatest concern for many people, and introduced the modified dental anxiety scale (MDAS). This modified scale was validated and published in Portuguese in 2003 (5).

Litt (6) reviewed the effects of anxiety on pain perception, and argued that in clinical situations of acute pain, anxiety and pain may be indistinguishable. Anxiety not only lowers the pain threshold, but actually leads to the perception of painless stimuli as painful.

It is widely accepted that psychological stress can produce effects in a variety of physiological systems that are similar to those produced by physical challenges due to activation of two stress response systems. These systems are the sympathetic or autonomic system and the hypothalamus-pituitary-adrenal axis (HPA axis) (7). Situations involving pain, anxiety and acute tissue injury increase the activity of the HPA axis which in turn enhances 
secretion of cortisol. Dental stimuli are also capable of provoking anxiety (8); however, few studies have investigated the association between dental anxiety and neuroendocrine changes. Salivary cortisol levels were determined in patients with diseases in which the aggravation of symptoms was related to increased levels of stress such as oral lichen planus (9), and recurrent aphthous stomatitis (10) and cortisol levels were highly associated with anxiety in both. Brand (11) evaluated anxiety and cortisol secretion before dental treatment sessions but found only correlation between dental anxiety and urine cortisol. Krueger et al. (12) investigated the use of the DAS in dental fear- induced neuroendocrine changes at two time points across an educational session and a treatment session during periodontal treatment. High anxiety patients showed a significant increase in salivary cortisol levels in educational sessions than low anxiety patients. However, the groups did not differ significantly in their salivary cortisol levels at the treatment session.

Cortisol is secreted from the adrenal cortex and dispersed to all body fluids, and can be detected in urine, serum or saliva (13). Salivary samples have the advantage of being non-invasive, stable at room temperature for up to a week, and they can be easily collected at home under stress-free conditions. Salivary cortisol represents only the bioactive fraction: cortisol not bound to cortisol-binding-globulin or other proteins, and its concentration does not depend on salivary flow rate (7).

A wide variety of patients visit the dental school emergency clinic and toothache is the major reason for seeking dental care. Setor de Urgência Odontológica, Faculdade de Odontologia, Universidade de São Paulo (SUO-FOUSP), offers free treatment to patients, 79\% with pain-related complaints and most of them from a lower social class. More than one in four new patients at this emergency clinic reported some level of dental anxiety (5).

People suffering from dental anxiety may have a different HPA axis response. Therefore, the aim of the present study was to investigate the possible relation of dental pain, self reported anxiety and salivary cortisol concentration in emergency dental patients prior to receiving treatment.

\section{Materials and Methods}

Subjects comprised adult patients ( $\geq 18$ years old) who sought dental treatment at SUO-FOUSP. As drugs such as antidepressants and those prescribed for Cushing's syndrome can impair the laboratory assessment of HPA axis activity (14), subjects with drug or alcohol abuse problems or prescribed medication intake were excluded from the study. The study protocol was approved by the ethics committee of Faculdade de Odontologia,
Universidade de São Paulo. All subjects were volunteers and written informed consent was obtained.

In order to control for the circadian rhythm of cortisol, all procedures were performed between 9 am and $11 \mathrm{am}$. Collection of information, recording and saliva sampling were done by a single female experimenter, before subjects received treatment.

Subjects were required to state their gender, age, educational level and family income. Anxiety levels were measured by MDAS (4). This consists of five multiplechoice items, each with five responses, the scale anchors being 1 , most calm and 5, most anxious. The cut-off point used was a score $\geq 16$, indicating that the patient felt somewhat anxious about dental treatment. A score of 19 or more indicated a highly anxious patient. A Brazilian version of the MDAS was used to assess dental anxiety; the reliability of this scale has already been demonstrated (5). Subjects were also asked if they had any previous traumatic experience at the dental clinic.

Subjects rated their pain intensity due to their dental complaint using the horizontal visual analogue scale (VAS), on a 100-mm line, labeled with anchors from "no pain" to "worst possible pain".

Unstimulated saliva was collected by asking the subjects to hold a soft cotton roll in the mouth until it was saturated with saliva. It was then transferred to a plastic tube (Salivette ${ }^{\mathrm{TM}}$, Sarstedt Inc., Nümbrecht, Germany). As saliva samples can be affected by food and smoking, saliva was collected $\geq 30 \mathrm{~min}$ after eating or smoking. All samples had to be of clear consistency, with low viscosity and without blood contamination. The obtained specimen was stored frozen until analysis. After thawing, saliva samples were centrifuged and were assayed in duplicate by a commercial enzyme immunoassay kit (Active ${ }^{\mathrm{TM}}$ Cortisol (Saliva) EIA DSL-10-67100, Diagnostic Systems Laboratories Inc., Webster, Texas, USA).

\section{Statistical analysis}

Chi-square tests $\left(\chi^{2}\right)$ with Yates' correction were used to compare the distribution of subjects in the categorical variables. Data of salivary cortisol levels are presented as mean values and standard deviation (SD). Salivary cortisol concentrations were log-transformed, which normalized distribution of cortisol measures. Student's $t$-tests were used to compare continuous variables. Correlations were explored by means of the Pearson correlation coefficient. Correlations involving MDAS scores were analyzed by means of Spearman correlation coefficient. Statistical significance was accepted at $P<0.05$. 


\section{Results}

A total of 76 patients were initially enrolled in this study. Three individuals did not meet the inclusion criteria: one patient revealed that he was using antidepressant medication only at the end of saliva collection, while the other two saliva samples presented blood contamination and discoloration. The dental anxiety; salivary cortisol levels and pain of 73 volunteers (39 women (53.4\%), 34 men $(46.6 \%)$ ) were analyzed. Their mean age was 38.22 (SD 13.06) years (38.46 (SD 11.89) for women and 37.94 (SD 14.46) for men), with a range of 18 to 76 years of age.

Table 1 summarizes the comparison between anxious and non anxious patients and associated factors. Dental anxiety was not related to demographic and socioeconomic characteristics or to salivary cortisol concentration. Patient's report of a previous traumatic event was significantly different between anxious and non anxious patients.

Table 2 shows the association between salivary cortisol concentration, gender, complaint of pain and dental anxiety. Pain was the only factor which caused a significant increase in salivary cortisol level.

No correlation was found between pain intensity measured by means of VAS scores (Fig. 1) and salivary cortisol levels $(\mathrm{r}($ Pearson $)=0.2157, P=0.0667)$. The mean VAS score for male patients was $29 \mathrm{~mm}$ (SD 34.14) and that for female patients was $41.81 \mathrm{~mm}$ (SD 38.23). There was no statistical difference between genders $(t, P=0.138)$.

Although there was a tendency for negative association between salivary cortisol and MDAS scores, no significant correlation was found (Fig. 2).

When salivary cortisol concentrations were distributed according to the age of the sample, there was a non-

Table 1 Sample distribution according to dental anxiety and associated factors $\left(n=73, \chi^{2}\right)$

\begin{tabular}{lll}
\hline Variables & Anxious & Non anxious \\
\hline Gender & $P=0.0977$ & \\
Female & 20 & 19 \\
$\quad$ Male & 10 & 24 \\
Age (years) & $P=0.0990^{*}$ & \\
$\quad<50$ & 26 & 36 \\
$\geq 50$ & 8 & 3 \\
Education (full years) & $P=0.9558$ & \\
$\quad<8$ & 15 & 23 \\
$\geq 8$ & 15 & 20 \\
Family income (Brazilian $\mathrm{MW}^{* *}$ ) & $P=0.2777$ & \\
$\leq 3$ & 22 & 25 \\
$>3$ & 8 & 18 \\
Previous traumatic experience & $P=0.0168$ & \\
$\quad$ No & 14 & 33 \\
$\quad$ Yes & 16 & 10 \\
\hline$*=$ Fisher's exact test & \\
$* *$ Brazilian minimum wages $=$ US\$165 & &
\end{tabular}

Table 2 Association between salivary cortisol concentrations, gender, pain and anxiety ( $n=$ $73, t)$

\begin{tabular}{llll}
\hline & $n$ & Mean salivary cortisol (nM) & SD $^{*}$ \\
\hline Gender & & $P=0.057$ & \\
$\quad$ Male & 34 & 2.023 & 1.3398 \\
$\quad$ Female & 39 & 1.2117 & 1.3015 \\
Pain & & $P=0.0042$ & \\
$\quad$ No & 32 & 1.0029 & 1.3066 \\
$\quad$ Yes & 41 & 2.1483 & 1.2888 \\
Dental anxiety & & $P=0.3775$ & \\
$\quad$ Non anxious & 43 & 1.7002 & 1.3545 \\
$\quad$ Anxious & 30 & 1.333 & 1.3054 \\
\hline * = Standard deviation & & &
\end{tabular}

* = Standard deviation 
significant negative association between these variables ( $\mathrm{r}$ $($ Pearson $)=-0.181, P=0.1253)$.

\section{Discussion}

Dental anxiety has been associated with avoidance of dental care (15), and affects not only general health, in the form of a sleep disturbance, but also social interactions and work performance (16). Emergency dental situations involve a high prevalence of fear and anxiety, and it is important for the dentist to be able to recognize such patients. In these situations, anxiety prevalence is much higher than the proportion reported in previous studies $(1,2)$.
Although many authors have shown that dental anxiety is more common in women than in men $(1,4,5,17,18)$, in the present study, there was no relation between gender and dental anxiety. This was in agreement with the findings of Locker et al. (2). However, in a previous study of patients attending the same dental service (5), a difference between men and women was observed. The difference in findings in the present study is probably due to a smaller sample size.

Vassend (1), Hakeberg et al. (15), Humphris et al. (4) and Milgrom et al. (18) reported that younger subjects were more anxious than older ones; however, in the present

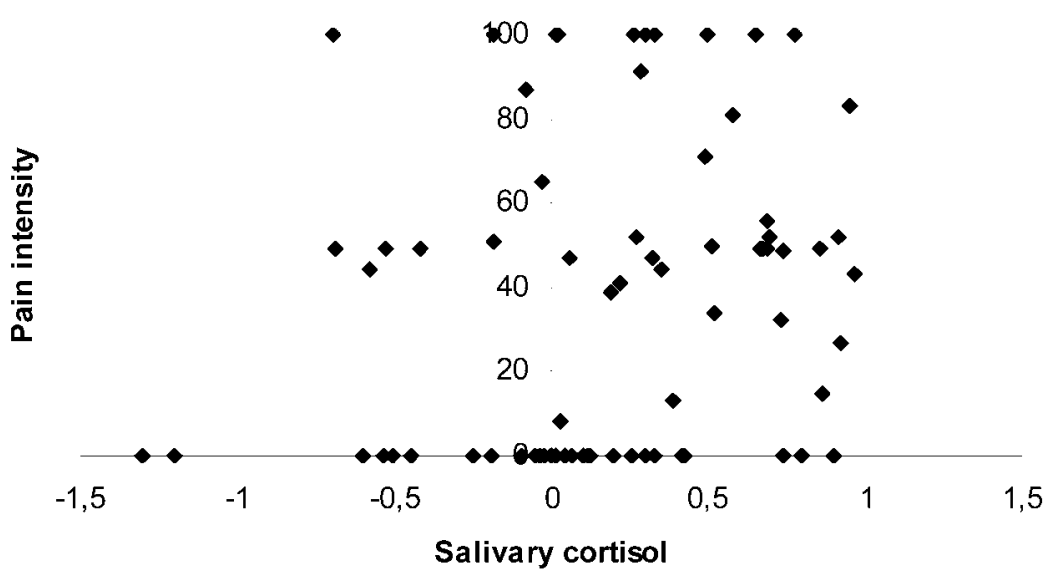

$r$ (Pearson $)=0.2157 P=0.0667$

Fig. 1 Correlation between salivary cortisol concentrations (logarithm transformed data) and pain intensity (VAS $\mathrm{mm}$ ).

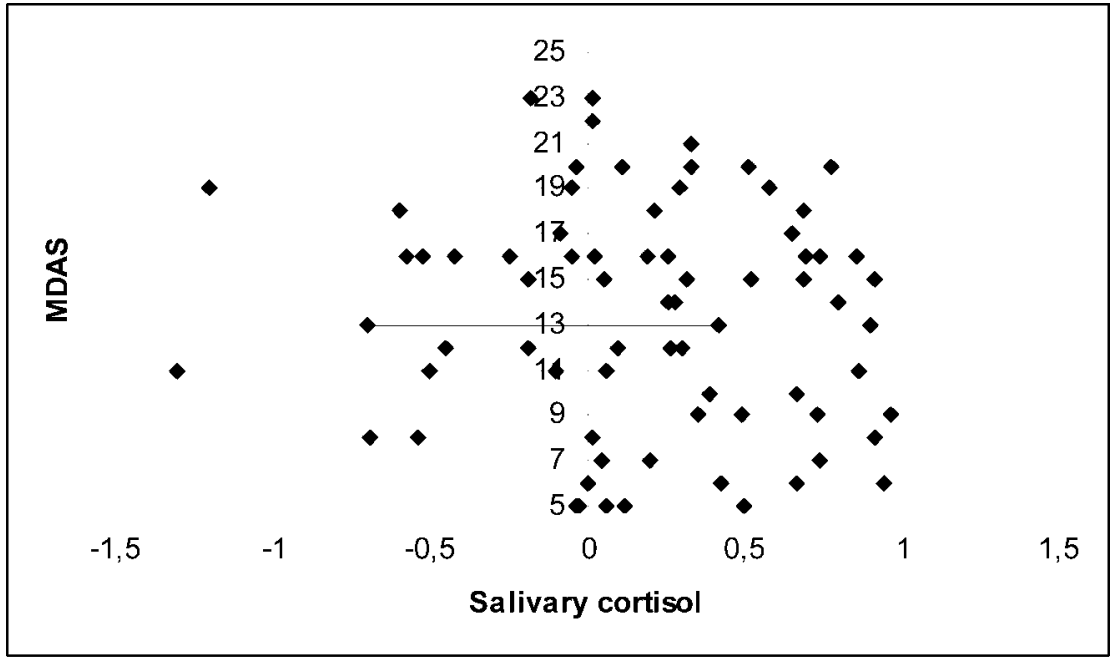

rs $($ Spearman $)=-0.0996 P=0.4016$

Fig. 2 Correlation between salivary cortisol concentrations (logarithm transformed data) and MDAS scores. 
study dental anxiety was not correlated with age. This may be due to the small number of patients aged $\geq 50$ who seek care at SUO-FOUSP; older subjects being more often edentulous. Therefore, the demand for emergency dental treatment is diminished among older patients as the main complaint of patients seeking emergency care is dental pain (5). Older patients also have often undergone more dental care in their lifetime, possibly facilitating habituation and enabling favorable reevaluation of the experience.

Differences in educational level and family income did not influence the dental anxiety level, as reported by Vassend (1), Kanegane et al. (5) and Hakeberg et al. (15). However, in the present study, persons with higher education and higher family income were underrepresented. When analyzing the results, patients were divided in order to improve distribution in these categories. This may have caused limitations when comparing these data. A greater number of patients with high level socio-economic backgrounds would help to determine if, and to what extent this non-response bias may have affected the results.

Although pain may occur during dental treatment, not everyone develops dental anxiety. An aversive experience may contribute, but particular psychological traits should also be associated (19). Oosterink et al. (9) found that individuals who had been exposed to distressing experiences in the past reported high levels of dental anxiety and other more distressing experiences. An important contributor of dental anxiety in patients receiving urgent care was the memory of a traumatic event (5). Even in this reduced sample, anxious individuals reported having experienced aversive dental episodes more than non anxious patients.

Emotional stress increases the activity of the HPA axis, which results in an enhanced secretion of adrenocortical hormones, and salivary cortisol is a reliable measure for assessment of its activity (7). This study investigated the effect of dental anxiety and emergency dental treatment on the HPA axis by measuring salivary cortisol. Dental anxiety alone was not enough to cause a reaction in the HPA axis and to produce a significant change in salivary cortisol concentration. As in a previous study (12), before a dental treatment session, no statistic difference was found between salivary cortisol levels of anxious and non anxious patients, although the mean salivary cortisol concentration of dentally anxious patients was lower than non anxious patients. In the majority of cases, dental emergencies are not life threatening, and patients do not go to the dentist immediately upon recognizing a dental problem (5). As these patients may avoid receiving the required dental care (15), a delayed dental visit can decrease the output of cortisol as a result of negative feedback on the HPA axis.
Individuals react to stressful events in different ways. Zimmer et al. and Kirschbaum et al. demonstrated that the pain is a stimulus that results in significant increases of salivary cortisol, and men show a larger cortisol response to a noxious stressor than women $(20,21)$. The present results are in concordance with these studies, in which pain produce different alterations in salivary cortisol concentration and indicate that gender may influence cortisol secretion.

In previous studies, response to the same noxious stimulus, men and women did not differ in VAS ratings of pain intensity (21). However, in this research, the mean VAS for pain intensity ratings for women was higher at the time of the interview; but no significant difference was found between the genders. This may be due to gender differences in perception and interpretation of the experience, and because the sensory and affective dimensions of pain are very closely identified in the minds of patients (6).

An important research area will be to investigate whether people with older age, higher education and higher income will display similar dental anxiety to their counterparts and its lack of influence on the HPA axis. Further studies concerning contribution of aversive experiences and particular psychological factors to the onset of dental anxiety are needed.

In conclusion, the occurrence of a traumatic dental experience is important for maintaining dental anxiety, and knowing how the experience was internalized it can help to provide a more effective treatment. Secondly, in emergency dental attendance, the variable pain has a greater risk of increasing salivary cortisol concentration.

\section{Acknowledgments}

The present study was supported by a grant from CAPES.

\section{References}

1. Vassend O (1993) Anxiety, pain and discomfort associated with dental treatment. Behav Res Ther 31, 659-666.

2. Locker D, Liddell A, Shapiro D (1999) Diagnostic categories of dental anxiety: a population-based study. Behav Res Ther 37, 25-37.

3. Corah NL (1969) Development of a dental anxiety scale. J Dent Res 48, 596.

4. Humphris GM, Morrison T, Lindsay SJ (1995) The modified dental anxiety scale: validation and United Kingdom norms. Community Dent Health 12, 143150.

5. Kanegane K, Penha SS, Borsatti MA, Rocha RG (2003) Dental anxiety in an emergency dental 
service. Rev Saúde Pública 37, 786-792. (in Portuguese)

6. Litt MD (1996) A model of pain and anxiety associated with acute stressors: distress in dental procedures. Behav Res Ther 34, 459-476.

7. King SL, Hegadoren KM (2002) Stress hormones: how do they measure up? Biol Res Nurs 4, 92-103.

8. Oosterink FM, de Jongh A, Aartman IH (2008) What are people afraid of during dental treatment? Anxiety-provoking capacity of 67 stimuli characteristic of the dental setting. Eur J Oral Sci $116,44-51$

9. Koray M, Dülger O, Ak G, Horasanli S, Üçok A, Tanyeri H, Badur S (2003) The evaluation of anxiety and salivary cortisol levels in patients with oral lichen planus. Oral Dis 9, 298-301.

10. Albanidou-Farmaki E, Poulopoulos AK, Epivatianos A, Farmakis K, Karamouzis M, Antoniades D (2008) Increased anxiety level and high salivary and serum cortisol concentrations in patients with recurrent aphthous stomatitis. Tohoku J Exp Med 214, 291296.

11. Brand HS (1999) Anxiety and cortisol excretion correlate prior to dental treatment. Int Dent J 49, 330336.

12. Krueger TH, Heller HW, Hauffa BP, Haake P, Exton MS, Schedlowski M (2005) The dental anxiety scale and effects of dental fear on salivary cortisol. Percept Mot Skills 100, 109-117.

13. Gozansky WS, Lynn JS, Laudenslager ML, Kohrt WM (2005) Salivary cortisol determined by enzyme immunoassay is preferable to serum total cortisol for assessment of dynamic hypothalamic-pituitaryadrenal axis activity. Clin Endocrinol 63, 336-341.

14. Ambrogio AG, Pecori Giraldi F, Cavagnini F (2008) Drugs and HPA axis. Pituitary 11, 219-229.

15. Hakeberg M, Berggren U, Carlsson SG (1992) Prevalence of dental anxiety in an adult population in a major urban area in Sweden. Community Dent Oral Epidemiol 20, 97-101.

16. Cohen SM, Fiske J, Newton JT (2000) The impact of dental anxiety on daily living. Br Dent J 189, 385390.

17. Liddell A, Locker D (1997) Gender and age differences in attitudes to dental pain and dental control. Community Dent Oral Epidemiol 25, 314318.

18. Milgrom P, Fiset L, Melnick S, Weinstein P (1988) The prevalence and practice management consequences of dental fear in a major US city. $\mathrm{J}$ Am Dent Assoc 116, 641-647.

19. Thomson WM, Locker D, Poulton R (2000) Incidence of dental anxiety in young adults in relation to dental treatment experience. Community Dent Oral Epidemiol 28, 289-294.

20. Zimmer C, Basler HD, Vedder H, Lautenbacher S (2003) Sex differences in cortisol response to noxious stress. Clin J Pain 49, 233-239.

21. Kirschbaum C, Wüst S, Hellhammer D (1992) Consistent sex differences in cortisol responses to psychological stress. Psychosom Med 54, 648-657. 